han sido traducidos a la mayoría de los idiomas de los países en desarrollo y están disponibles para otras investigaciones, especialmente en poblaciones con baja escolaridad. Asimismo, se prestan para realizar comparaciones válidas entre poblaciones de diferentes países y con distintas culturas. (Prince M, Acosta D, Chiu H, Scazufca M, Varghese M. Dementia diagnosis in developing countries: a crosscultural validation study. Lancet. 2003;361(9361): 909-17.)

\section{La inmunidad provoca el aumento de la virulencia en un modelo experimental de malaria}

Diversas investigaciones han revelado la presencia de cambios genéticos que se asocian con la evolución de la virulencia en organismos patógenos, es decir, con el aumento o la disminución de su capacidad para causar daños al organismo hospedero. Numerosos mecanismos pueden facilitar la adquisición de rasgos genéticos que modifican la virulencia de los organismos patógenos. Por ejemplo, la inmunidad del hospedero contra el patógeno - ya sea adquirida de forma natural o mediante la vacunación- puede evitar que aquel muera, lo cual le permite al patógeno vivir más tiempo y tener más oportunidad de evolucionar. De esta forma, en poblaciones de personas con un alto grado de inmunidad es factible que surjan cepas patógenas más virulentas que las halladas en poblaciones de personas sin inmunidad.

La inmunidad de la población también puede ejercer una presión selectiva dentro del propio hospedero, como resultado de una competencia entre organismos patógenos con diferentes grados de virulencia. Lamentablemente, no se conocen los mecanismos de esta selección.

En este estudio se utilizó un modelo experimental de malaria murina (causada por Plasmodium chabaudi) para determinar si la presión selectiva ejercida por la inmunidad lleva al agente patógeno a adquirir mayor virulencia. En el estudio se utilizaron dos líneas progenitoras de $P$. chabaudi (una virulenta y otra inocua) y ratones hembras C57BI/6J de 7 a 10 semanas de edad. Cinco sublíneas de cada línea progenitora del parásito se pasaron repetidamente por ratonas inmunizadas y sin inmunizar, con lo que se establecieron 20 sublíneas en total. Las pasadas se realizaron mediante la inoculación de
$0,1 \mathrm{~mL}$ de sangre diluida que contenía $5 \times 10^{5}$ parásitos obtenida de ratonas donantes que habían sido infectadas siete días antes, es decir, se obvió el paso del parásito por el mosquito vector. Después de 20 pasadas se compararon el grado de virulencia y la capacidad de transmisión de las sublíneas parasitarias que habían pasado por las ratonas inmunizadas y las de las sublíneas parasitarias que habían pasado por las ratonas sin inmunizar. Seguidamente se probó si el aumento de la virulencia se mantenía cuando el parásito era transmitido por un mosquito.

Tanto las sublíneas de parásitos sometidas a 20 pasadas sucesivas en las ratonas inmunizadas como las pasadas por las ratonas sin inmunizar mostraron una mayor virulencia que la población parasitaria progenitora. El aumento de la virulencia fue mayor en las sublíneas derivadas de las ratonas inmunizadas. Cuando después de las 20 pasadas los parásitos se transmitieron mediante mosquitos, se observó una reducción de la virulencia en todas las sublíneas, pero las sublíneas obtenidas mediante pases en ratonas inmunizadas mantuvieron una mayor virulencia que las obtenidas en las ratonas sin inmunizar.

Estos resultados demuestran que la inmunidad del huésped actúa como una poderosa fuerza selectiva dentro del hospedero y que esta presión refuerza la virulencia del parásito infectante. Sin embargo, se requieren investigaciones adicionales que expliquen el mecanismo que provoca estos cambios. Como la inmunización evita la muerte del hospedero, las variantes más virulentas del parásito se ven favorecidas por la presión selectiva interna, lo que puede provocar la diseminación de líneas más virulentas en poblaciones de hospederos inmunizados.

La presión selectiva ejercida por la inmunidad del huésped aceleró en las líneas de Plasmodium la adquisición de mayor virulencia. Este hecho, unido a la conocida capacidad de este parásito de adaptarse rápidamente a nuevas condiciones de laboratorio y al efecto de la inmunización con vacunas específicas contra diferentes variantes, confirman la necesidad de mantener un seguimiento constante de la virulencia de Plasmodium en poblaciones vacunadas contra la malaria. (Mackinnon M, Read AF. Immunity promotes virulence evolution in a malaria model. PLoS Biol. 2004;2(9):e230. Hallado en:http://www.plosbiology.org/plosonline/ ?request $=$ get - document $\&$ doi $=10.1371 /$ journal . pbio. 0020230. Acceso el 23 de junio de 2004.) 